\title{
Association of Diabetic Retinopathy, Albuminuria and CKD in Patients with Type 2 Diabetes Mellitus
}

\author{
Authors \\ Dr Annapurna Kumar ${ }^{1}$, Dr Ravish Vaishnav ${ }^{2}$ \\ ${ }^{1}$ Sri Venkateshwaraa Medical, College Hospital and Research, Centre, Pondicherry \\ ${ }^{2}$ Consultant Ophthalmologist \\ Corresponding Author \\ Dr Annapurna Kumar \\ Email: annapurna200@gmail.com,9442395599
}

\begin{abstract}
Background: Retinopathy and renal involvement are two of the major microvascular complications of diabetes mellitus. Recent reports challenge the traditional view of renal involvement progressing from increased glomerular filtation to progressively increasing levels of albuminuria that culminate in chronic kidney disease, and have found that retinopathy and albuminuria may not always co-exist. This study was undertaken to understand the relationship between retinopathy, albuminuria and chronic kidney disease in patients with type 2 diabetes mellitus.

Methods: One hundred patients with type 2 diabetes mellitus attending the diabetic clinic were evaluated for retinopathy and renal parameters that included a 24 urinary albumin excretion, serum creatinine and evidence of chronic kidney disease based on the estimated glomerular filtration rate.

Results: 67 patients were found to have retinopathy, out of which 12 (17.9\%) had no evidence of any abnormal renal parameters, 25 (37.3\%) had only abnormal albuminuria (microalbuminuria or macralbuminuria), one (1.5\%) had evidence of CKD, and 29 (43.3\%) had both albuminuria and CKD. 16 patients with CKD did not have any evidence of retinopathy.

Conclusion: There was a statistically significant association between the presence and severity of retinopathy, and abnormal urinary excretion of albumin. A similar association between retinopathy and CKD was not seen.

Keywords: Diabetic retinopathy, Microalbuminuria, Macroalbuminuria, Normoalbuminuria, Chronic kidney disease, Estimated glomerular filtration rate.
\end{abstract}

\section{Introduction}

Diabetes mellitus is of worldwide occurrence. The prevalence of diabetes mellitus in India in a recent large nation-wide study was found to be $7.3 \%$, and the incidence of pre diabetes to be $10.3 \% .^{(1)}$ Diabetic retinopathy, one of the microvascular complications of diabetes, is one of the leading causes of blindness in working age adults., ${ }^{(2)}$ affecting varying proportions of the population with significant ethnic variations. ${ }^{(3)}$

Renal involvement is the other major microvascular complication of diabetes. Historically, the description of renal involvement in diabetes mellitus has followed a natural course of glomerular hyper filtration, and progressively increasing levels of urinary albumin excretion, 
eventually leading to a decline in the glomerular filtration rate which eventually leads to end stage renal disease. Retinal involvement in diabetes is usually closely associated with albuminuria, with more severe forms associated with greater degrees of albuminuria. ${ }^{(4)}$

However recent studies have disputed this traditional thinking, and there have been reports of albuminuria and GFR (glomerular filtration rate) not invariably following the clear cut progression previously described, and of retinopathy that doesn't always parallel the albuminuria and declining renal function. ${ }^{(5,6)}$ This is especially pronounced in type 2 diabetes mellitus where a non-albuminuric pathway to chronic kidney disease has been found in many studies. ${ }^{(7-10)}$.

There are few reports in India which have studied in detail the association between retinopathy, albuminuria and GFR. This study was conducted to determine the relationship between these there parameters in a tertiary care hospital in South India.

\section{Materials and Methods}

This cross sectional study was undertaken in eligible patients after obtaining clearance from the Institutional Ethics Committee, and conformed to the tenets of the declaration of Helsinki. The study was conducted in patients with type 2 diabetes mellitus who were seen in the diabetic clinic. Eligible patients who gave written informed consent were taken up for the study. Patients suffering from uncontrolled hypertension, severe vascular disease, treatment with systemic steroids orany other systemic diseases that could lead to renal involvement were excluded from the study. Patients with high myopia and other ocular comorbidities (excluding other refractive errors) were also excluded from the study.

Each patient was then subjected to a complete workup which included a full medical history and a thorough ophthalmologic examination. Fundus examination with indirect ophthalmoscopy and slit lamp indirect ophthalmoscopy was done to assess and grade retinopathy. Retinopathy was classified as mild non-proliferative diabetic retinopathy (NPDR), moderate (NPDR), severe (NPDR), proliferative diabetic retinopathy (PDR) and advanced diabetic eye disease (ADED) based on ETDRS (Early Treatment Diabetic Retinopathy Study) guidelines. If there was a difference in retinopathy between the two eyes, the grade of retinopathy in the worse eye was taken for analysis.

The laboratory parameters taken for assessment included fasting blood sugar (FBS), post-prandial blood sugar (PPBS), serum hemoglobin, HbA1c levels, serum urea,serum creatinine and 24 hour urine albumin levels. Based on the 24 hour urine albumin results, patients were graded as having normoalbuminuria, microalbuminuria or macroalbuminuria, where, normoalbuminuria indicated a 24 hour urine albumin excretion value of $<30 \mathrm{mg}$ per day, 30-300 mg per day for microalbuminuria, and $>300 \mathrm{mg}$ per day for macroalbuminuria. ${ }^{(7)}$

The estimated glomerular filtration rate (eGFR) was calculated using the Chronic Kidney Disease Epidemiology Collaboration (CKD-EPI) equation. (11) CKD (chronic kidney disease) was definedas an eGFR of $<60 \mathrm{ml} / \mathrm{min} / 1.73 \quad \mathrm{~m}^{2 .(7)}$ The relationship of diabetic retinopathy to albuminuria and CKD was studied.

Statistical Analysis: IBM SPSS 16 was used for statistical analysis. Descriptive parameters were presented as mean \pm standard deviation and qualitative variables such as gender, presence and grades of retinopathy, dry eye, diminished corneal sensation were expressed as proportions. Association of retinopathy with other variables was analyzed using Chi-square test of significance.A p value of $<0.05$ was considered significant.

\section{Results}

Of the 100 patients with type 2 diabetes mellitus who were found eligible to participate in the study, 51 were males and 49 were females, ranging in age from $40-83$ years with a mean age of 56.79 years and a standard deviation of 9.61. $67(67 \%)$ patients had some form of retinopathy. Out of these, 17 (25.4\%) had mild NPDR (non 
proliferative diabetic retinopathy), $16(23.9 \%)$ had moderate NPDR, 15 (22.4\%) had severe NPDR, 11 (16.4\%) had PDR (proliferative diabetic retinopathy), and $8(11.9 \%)$ had ADED (advanced diabetic eye disease). Out of 67 patients with retinopathy, $12(17.9 \%)$ had no evidence of any abnormal renal parameters, 25 (37.3\%) had only abnormal albuminuria (microalbuminuria or macralbuminuria), one $(1.5 \%)$ had only a diminished eGFR, and 29 (43.3\%) had both an albuminuria and a diminished eGFR. There was a statistically significant association between the presence of retinopathy and the presence of abnormal urinary albumin $(\mathrm{p}=0.02)$ (Table 1$)$.
Table 1: Association of retinopathy with albuminuria

\begin{tabular}{|c|c|c|c|}
\hline & \multicolumn{2}{|c|}{ Retinopathy } & \multirow{2}{*}{ p value } \\
\cline { 1 - 3 } Albuminuria & $\begin{array}{c}\text { Present } \\
\text { N (\%) }\end{array}$ & $\begin{array}{c}\text { Absent } \\
\text { N (\%) }\end{array}$ & \\
\hline Normoalbuminuria & $13(19.4)$ & $13(39.4)$ & \multirow{2}{*}{0.02} \\
\cline { 1 - 3 } Microalbuminuria & $32(47.8)$ & $16(48.5)$ & \\
\cline { 1 - 3 } Macroalbuminuria & $22(32.8)$ & $4(12.1)$ & \\
\hline
\end{tabular}

The proportion of albuminuria in the various stages of retinopathy is shown in Figure 1. Only one patient out of the 34 patients with advanced retinopathy (which included severe NPDR, PDR and ADED) had normoalbuminuria (Figure 2)

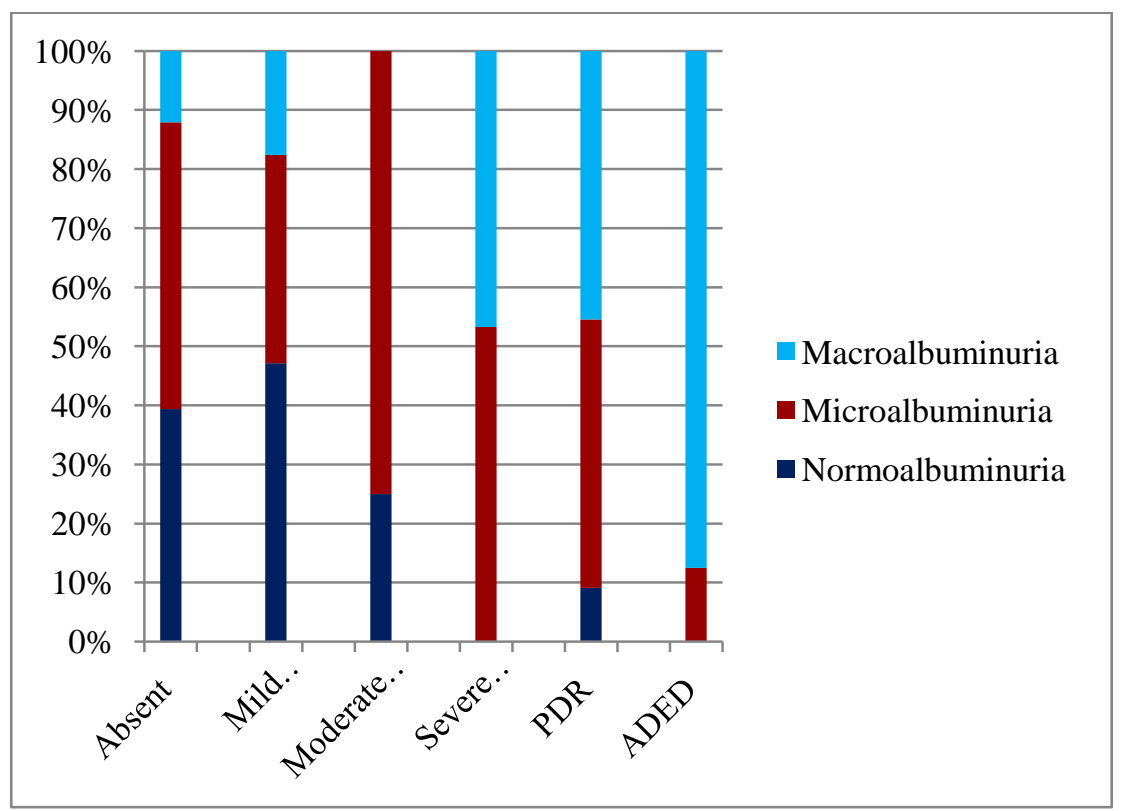

Figure 1: Association of albuminuria in various stages of retinopathy Mild - mild NPDR, Moderate - moderate NPDR, Severe- severe NPDR.

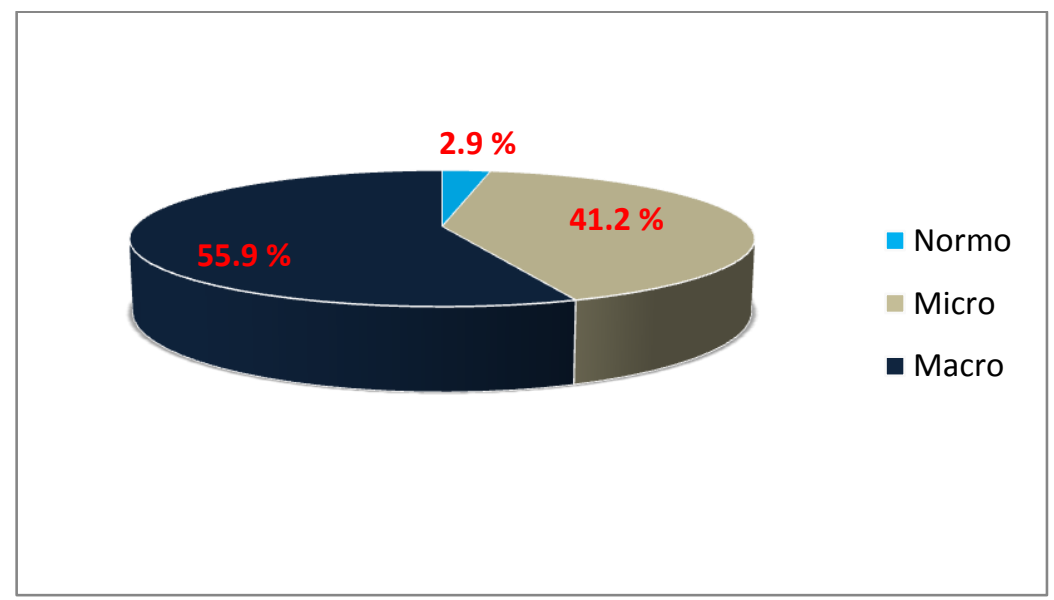

Figure 2: Percentage of urinary albumin levels in patients with advanced retinopathy Normo-Normoalbuminuria, Micro-Microalbuminuria, Macro-Macroalbuminuria 
Comparing the relationship between retinopathy and chronic kidney disease (CKD), there was no statistically significant association between presence of retinopathy and eGFR. $48.5 \%$ of those patients with no retinopathy had evidence of CKD whereas only $44.8 \%$ of patients with any form of retinopathy had CKD. The percentage of patients with various types of retinopathy associated with a diminished eGFR is given in Table 2

Table 2: Association of retinopathy with reduced eGFR.

\section{Number of patients with reduced eGFR \\ Retinopathy (CKD)}

Percentage

\begin{tabular}{|c|c|c|}
\hline $\begin{array}{l}\text { Absent } \\
(n=33)\end{array}$ & 16 & 48.5 \\
\hline $\begin{array}{l}\text { Mild NPDR } \\
(\mathrm{n}=17)\end{array}$ & 4 & 23.5 \\
\hline $\begin{array}{l}\text { Moderate } \\
\text { NPDR } \\
(n=16)\end{array}$ & 4 & 25 \\
\hline $\begin{array}{l}\text { Severe NPDR } \\
(n=15)\end{array}$ & 8 & 53.3 \\
\hline $\begin{array}{l}\text { PDR } \\
(n=11)\end{array}$ & 6 & 54.5 \\
\hline $\begin{array}{l}\text { ADED } \\
(\mathrm{n}=8)\end{array}$ & 8 & 100 \\
\hline
\end{tabular}

Fifty four patients had serum creatinine levels of one or less. 30 of them $(55.6 \%)$ had microalbuminuria and four patients had macroalbuminuria. 14 patients with normal serum creatining levels and absence of retinopathy had microalbuminuria, and one patient had macroalbuminuria.

\section{Discussion}

We found a significant association between the presence of retinopathy and abnormal urinary excretion (either microalbuminuria or macroalbuminuria) in patients with type 2 diabetes mellitus. All patients with advanced retinopathy (except for one) had abnormal urinary albumin excretion, and more than half of them (55.9\%) had macroalbuminuria, which, signals the stage of overt nephropathy in staging of diabetic nephropathy. ${ }^{(4)}$ Other studies have found this relationship between retinopathy and albuminuria as well. ${ }^{(12-15)}$

On the other hand, there was no statistically significant association of retinopathy with reduced eGFR. In our study, a greater percentage of patients with no retinopathy had CKD as compared to patients with any form of retinopathy. $22(47.8 \%)$ of patients with CKD had advanced retinopathy. 17 of these patients (77.3\%) had macroalbuminuria. This supports the premise that the presence and severity of retinopathy is more related to the presence and severity of albuminuria rather than to the eGFR.

Sabanayagamet all also found a similar association between a reduced eGFR and retinopathy that was related to albuminuria. This relationship has been found in other studies as well. ${ }^{(16-19)}$

However, not all studies have found this correlation between retinopathy and albuminuria. In the NHANES study, Kramer et al have found $30 \%$ of patients with chronic renal insufficiency with neither retinopathy nor albuminuria; they did not find any correlation between retinopathy and albuminuria either.

Even in studies that showed a relationship between albuminuria and retinopathy, there were still cases of retinopathy occurring in patients with normoalbuminuria. ${ }^{(13,15)} 50 \%$ of patients in our study with normoalbuminuria had retinopathy. More than half of those with retinopathy had mild NPDR, and one had proliferative diabetic retinopathy. Also, many patients with no retinopathy were found to have advanced renal disease. This suggests that all diabetic patients need to undergo a thorough ophthalmic and renal parameter evaluation in order to detect preventable or treatable complications of diabetes. This is especially relevant as patients seen in the outpatient diabetic or medicine clinic may not always be screened for retinopathy ${ }^{(20)}$. Absence 
of microalbuminuria may be responsible for this complacence. ${ }^{(15)}$

Conversely, ophthalmologists should not be content with screening their patients with serum creatinine alone, as, $15 \%$ of the patients in this study had normal serum creatinine along with an absence of retinopathy, but were positive for abnormal urinary albumin.

The results of our study may be limited by the cross sectional design. However, they correlate well with other cross sectional studies ${ }^{(12-14)}$

\section{Conclusion}

There is a statistically significant association between diabetic retinopathy and albuminuria in patients with type 2 diabetes mellitus. However, this relationship is not absolute, and individual patients still need to be screened for both retinopathy and renal disease, as the two are not always co-existent.

\section{References}

1. Anjana RM, Deepa M, Pradeepa R, Mahanta J, Narain K, Das HK, et al. Prevalence of diabetes and prediabetes in 15 states of India: results from the ICMRINDIAB population-based cross-sectional study. Lancet Diabetes Endocrinol. 2017;5:585-96.

2. Gerstein HC, Ambrosius WT, Danis R, Ismail-Beigi F, Cushman W, Calles J, et al. Diabetic Retinopathy, Its Progression, and Incident Cardiovascular Events in the ACCORD Trial. Diabetes Care. 2013;36:1266-71.

3. Yau JWY, Rogers SL, Kawasaki R, Lamoureux EL, Kowalski JW, Bek T, et al. Global Prevalence and Major Risk Factors of Diabetic Retinopathy. Diabetes Care. 2012;35:556-64.

4. Pugliese G. Updating the natural history of diabetic nephropathy. Acta Diabetol. 2014;51:905-15.

5. Kramer HJ, Nguyen QD, Curhan G, Hsu C-Y. Renal insufficiency in the absence of albuminuria and retinopathy among adults with type 2 diabetes mellitus. JAMA. 2003;289:3273-7.

6. Retnakaran R, Cull CA, Thorne KI, Adler AI, Holman RR. Risk Factors for Renal Dysfunction in Type 2 Diabetes. Diabetes. 2006;55:1832-9.

7. Parving $\mathrm{H}-\mathrm{H}$, Persson $\mathrm{F}$, Rossing $\mathrm{P}$. Microalbuminuria: a parameter that has changed diabetes care. Diabetes Res Clin Pract. 2015;107:1-8.

8. Reutens AT. Epidemiology of diabetic kidney disease. Med Clin North Am. 2013;97:1-18.

9. Boronat M, García-Cantón C, Quevedo V, Lorenzo DL, López-Ríos L, Batista F, et al. Non-albuminuric renal disease among subjects with advanced stages of chronic kidney failure related to type 2 diabetes mellitus. Ren Fail. 2014;36:166-70.

10. Sabanayagam C, Foo VHX, Ikram MK, Huang H, Lim SC, Lamoureux EL, et al. Is chronic kidney disease associated with diabetic retinopathy in Asian adults? J Diabetes. 2014;6:556-63.

11. Inker LA, Levey AS. Pro: Estimating GFR using the chronic kidney disease epidemiology collaboration (CKD-EPI) 2009 creatinine equation: the time for change is now. Nephrol Dial Transplant. 2013 Jun 1;28:1390-6.

12. Sharma A, Sharma R, Ankur, Paul J, Tyagi A, Khari S, et al. A Study of Microalbuminuria Levels among Patients with Type 2 Diabetic Complications in Western U.P. Int $\mathbf{J}$ Contemp Med. 2017;5:84.

13. Rani PK, Raman R, Gupta A, Pal SS, Kulothungan V, Sharma T. Albuminuria and Diabetic Retinopathy in Type 2 Diabetes Mellitus Sankara Nethralaya Diabetic Retinopathy Epidemiology And Molecular Genetic Study (SN-DREAMS, report 12). Diabetol Metab Syndr. 2011 May 25;3:9. 
14. Manaviat MR, Afkhami M, Shoja MR. Retinopathy and microalbuminuria in type II diabetic patients. BMC Ophthalmol. 2004;4:9.

15. Karoli R, Fatima J, Shukla V, Garg P, Ali A. Predictors of diabetic retinopathy in patients with type 2 diabetes who have normoalbuminuria. Ann Med Health Sci Res. 2013;3:536-40.

16. Lampropoulou I-T, Stangou M, Papagianni A, Didangelos $\mathrm{T}$, Iliadis $\mathrm{F}$, Efstratiadis G. TNF- $\alpha$ and microalbuminuria in patients with type 2 diabetes mellitus. J Diabetes Res. 2014; 7

17. Penno G, Solini A, Zoppini G, Orsi E, Zerbini G, Trevisan R, et al. Rate and Determinants of Association Between Advanced Retinopathy and Chronic Kidney Disease in Patients With Type 2 Diabetes. Diabetes Care. 2012;35:231723.

18. Gall M-A, Hougaard P, Borch-Johnsen K, Parving H-H. Risk factors for development of incipient and overt diabetic nephropathy in patients with non-insulin dependent diabetes mellitus: prospective, observational study. BMJ. 1997 Mar 15;314:783.

19. Moriya T, Tanaka S, Kawasaki R, Ohashi Y, Akanuma Y, Yamada N, et al. Diabetic Retinopathy and microalbuminuria can predict macroalbuminuria and renal function decline in Japanese type 2 diabetic patients. Diabetes Care. 2013;36:2803-9.

20. MacLennan PA, McGwin G, Heckemeyer C, Lolley VR, Hullett S, Saaddine J, et al. Eye Care Use Among a High-Risk Diabetic Population Seen in a Public Hospital's Clinics. JAMA Ophthalmol. 2014;132:162-7. 